\title{
DNA-based diagnosis of malignant osteopetrosis by whole-genome scan using a single-nucleotide polymorphism microarray: standardization of molecular investigations of genetic diseases due to consanguinity
}

\author{
Ching-Wan Lam $\cdot$ Sui-Fan Tong $\cdot$ Keong Wong $\cdot$ \\ Y. F. Luo $\cdot$ Hoi-Yin Tang $\cdot$ Shau-Yin Ha \\ Michael Ho-Ming Chan \\ Received: 3 June 2006/ Accepted: 21 September 2006/Published online: 11 October 2006 \\ (C) The Japan Society of Human Genetics and Springer-Verlag 2006
}

\begin{abstract}
Malignant osteopetrosis, a severe disease causing early infantile death in humans, is caused by mutations in the TCIRG1, CLCN7, or OSTM1 genes. We have established the molecular basis of malignant osteopetrosis in a Chinese family by means of wholegenome scans based on high-density single-nucleotide polymorphism (SNP) microarrays. Because the parents were consanguineous, the disease-causing locus should be located in an autozygous chromosomal region. Mapping revealed that among the three possible causal loci, only the CLCN7 gene was located in an autozygous region. Mutational analysis of the CLCN7 gene showed that the proband was homozygous for a novel missense mutation, p.I261F. p.I261 is located in helix F of the chloride channel, near a critical site for gating of the channel. This mapping study prepares the ground for future mutation studies by decreasing the burden of
\end{abstract}

C.-W. Lam $(\varangle) \cdot$ S.-F. Tong · M. H.-M. Chan

Department of Chemical Pathology,

The Chinese University of Hong Kong,

Prince of Wales Hospital,

Hong Kong, China

e-mail: ching-wanlam@cuhk.edu.hk

K. Wong · Y. F. Luo

Department of Obstetrics and Gynecology,

Centro Hospitalar Conde S. Januario,

Macau, China

H.-Y. Tang

Prenatal Diagnosis and Counseling Department,

Tsan Yuk Hospital, Hong Kong, China

S.-Y. Ha

Department of Pediatrics and Adolescent Medicine,

The University of Hong Kong, Queen Mary Hospital,

Hong Kong, China completely sequencing all possible loci for this disease. This approach can be used to standardize molecular investigations of genetic diseases due to consanguinity to a whole-genome scan and subsequent sequencing of the mapped disease gene.

Keywords Homozygosity mapping · Malignant osteopetrosis $\cdot$ Single-nucleotide polymorphism microarray $\cdot$ Whole-genome scan

\section{Introduction}

Malignant osteopetrosis (OPTB1; OMIM 259700) or autosomal recessive osteopetrosis (ARO) is characterized by severe osteosclerosis, pathologic fractures, hepatosplenomegaly, and pancytopenia due to an osteoclast dysfunction that results in inadequate bone resorption. ARO is a severe disease, causing early infantile death if bone marrow is not successfully transplanted. The malignant course of the disease and the often unfavorable outcome of bone marrow transplantation (BMT) make prenatal diagnosis very important. However, prenatal ultrasound examination can be normal for a fetus with osteopetrosis (Shalev et al. 2001). Therefore, molecular analysis provides the best method for making a reliable prenatal diagnosis.

ARO can result from mutations in the TCIRG1 gene (T-cell, immune regulator 1 , ATPase, $\mathrm{H}+$ transporting, lysosomal V0 protein a isoform 3) (Frattini et al. 2000), CLCN7 gene (chloride channel 7) (Kornak et al. 2001), or OSTM1 gene (osteopetrosis-associated transmembrane protein 1) (Chalhoub et al. 2003). Most ARO cases have been ascribed to mutations in the TCIRG1 gene, with only a few cases 
attributed to mutations in the CLCN7 gene (Cleiren et al. 2001). For prioritization of mutational analysis of the three possible disease-causing genes, we have performed genetic mapping of the ARO loci of a Chinese family by whole-genome scan using highdensity single-nucleotide microarrays.

\section{Materials and methods}

\section{Case presentation}

A Chinese female baby (Fig. 1; II:3) was diagnosed as suffering from malignant infantile osteopetrosis at 3 weeks of age. She was delivered by normal spontaneous vaginal delivery. There were no abnormal findings during the antenatal period; in particular, both the ultrasonographic features and intra-uterine growth were normal. Three months after the baby was born, the first set of skeletal radiographs was taken. Increases in bone density in the cervical spine and the epiphyseal growth plates of the long bones were suggestive of early osteopetrotic changes. The patient was referred to us for BMT. After BMT, the blood count, bone marrow, and chimerism showed good engraftment; however, the X-rays still showed generalized increased bone density, and ophthalmologic examination showed pale optic discs. No pigmentary retinopathy was noted.

The proband has two elder brothers (II:1, II:2) and the parents (I:1, I:2) are first cousins. The first brother (II:1) was diagnosed to suffer from the same condition at the age of 14 months, when he presented unexpectedly with a sudden development of generalized petechiae, anemia, thrombocytopenia, and hepatosplenomegaly. Trephine biopsy revealed typical histological features of osteopetrosis, including increased trabecular size and matrix thickening with abnormal medullary bone formation and narrowing of the

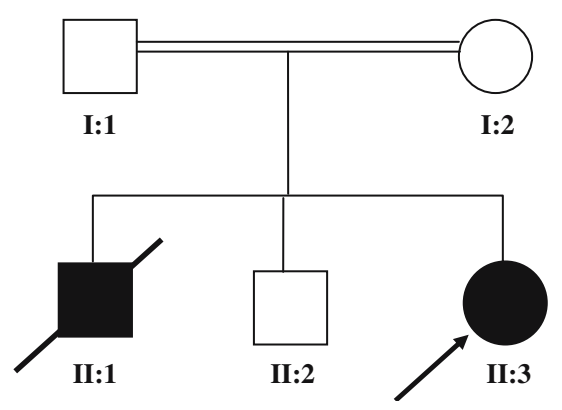

Fig. 1 Pedigree of the family. Filled square and circle indicate siblings with malignant infantile osteopetrosis medullary space. A skeletal survey confirmed an increase in general bone density. Early signs of right optic nerve atrophy were subsequently detected. He died 9 months later due to respiratory failure as a result of bronchiolitis obliterans following allogenic mismatched BMT performed with the mother as the donor. The second brother (II2) was phenotypically normal.

\section{DNA extraction}

Genomic DNA of the proband, the parents, and the two brothers was extracted from peripheral blood samples with a QIAamp blood kit (Qiagen, Hilden, Germany) according to the manufacturer's instruction. Participants gave their written informed consent. The study was conducted according to the Declaration of Helsinki Principles.

HuSNP probe array and HuSNP mapping assay

In the two siblings (II1 and II3) with ARO, we examined the homozygosity of single-nucleotide polymorphisms (SNP) flanking all three ARO loci using a whole-genome scan with HuSNP probe arrays (Affyetrix, Santa Clara, Calif.). Because the parents are consanguineous, the two disease-causing chromosomal regions, one from each parent, are identical-by-descent and, consequently, the disease-causing locus of this family should fall in a chromosomal region marked by homozygous SNPs. SNP genotyping was performed according to the HuSNP protocol supplied by Affymetrix. HuSNP probe arrays are manufactured using technology that combines photolithographic methods and combinatorial chemistry. Tens to hundreds of thousands of different oligonucleotide probes are synthesized in a $0.81 \times 0.81-\mathrm{cm}$ area on each HuSNP probe array. With a median marker gap size of $1.2 \mathrm{cM}, 1308$ fully mapped markers are evenly distributed across the human genome (Fan et al. 2000).

Briefly, starting with $120 \mathrm{ng}$ of genomic DNA, a set of 24 simultaneously run multiplex PCRs amplify the human SNPs represented in the GeneChip HuSNP mapping assay. The amplified SNPs are further amplified and concomitantly labeled using biotinylated primers in a second set of 24 simultaneously run labeling PCRs. The biotinylated PCR products are then pooled, concentrated, and prepared for hybridization. The biotinylated amplification products, which reflect the bi-allelic genotype (typed as A or B) in the sample DNA, are hybridized to the GeneChip HuSNP probe arrays during an overnight incubation at $44^{\circ} \mathrm{C}$ in 
the GeneChip hybridization oven. The following day, the probe arrays are thoroughly washed and stained with a complex of streptavidin-phycoerythrin and biotinylated anti-streptavidin immunoglobulin $\mathrm{G}$ antibody. The automated wash and stain procedures are run on the GeneChip fluidics station 400 under the control of Affymetrix Microarray Suite software ver. 5.0. The stained probe arrays are scanned in an Agilent GeneArray scanner, also under the control of Affymetrix Microarray Suite software ver. 5.0. Each probe array is scanned twice to capture the light emitted at wavelengths of 530 and $570 \mathrm{~nm}$, generating two scan image files. The Affymetrix Microarray Suite software 5.0 processes the two scan images to calculate the signal intensities on the probe array. Genotype calling is performed by GeNOTYPE VIEWER ver. 1.0 (Affymetrix). The SNP genotyping assay is fully automated, and the entire parallel assay can be completed within 2 days.

Mutational analysis

All of the exons and intro-exon boundaries of the CLCN7 gene were amplified by PCR as described (Cleiren et al. 2001). PCR products were purified by Microspin S-300 HR columns (GE Healthcare, Uppsala, Sweden), and both strands were sequenced using BigDyeDeoxy terminator cycle sequencing reagents according to the manufacturer's instructions (Applied Biosystems, Foster City, Calif.). Products of the sequencing reactions were purified by Centri-Sep spin columns (Princeton Separations, Adelphia, N.J.). Purified sequencing fragments were separated by capillary electrophoresis and detected via laser-induced fluorescence on an ABI Prism 3100 genetic analyzer (Applied Biosystems).

\section{Results}

According to the National Center for Biotechnology Information build 34 human genome map, the TCIRG1 gene is located on chromosome 11q13.2, the CLCN7 gene on chromosome 16p13.3, and the OSTM1 gene on chromosome 6q21. For these three loci, we found that only chromosome $16 \mathrm{p}$, harboring the CLCN7 gene, was marked by consecutive homozygous markers. The homozygous region was shared by II:1 and II:3 and spanned a chromosomal region of $57 \mathrm{cM}$ (data not shown). Thus, the $C L C N 7$ gene is likely to be the disease-causing gene in this family and subsequently selected for mutation screening. Using direct DNA sequencing, the two siblings (II:1 and II:3) with ARO were found to be homozygous for a c.781A $>$ T mutation in exon 9, which changes codon 261 from ATC to TTC as, for example, in I261F (Fig. 2). The parents (I:1 and I:2) and the normal sibling (II:2) were all heterozygous for p.I261F. p.I261F is a non-conservative change among various chloride channels. In search of a control, we screened 50 normal Chinese individuals for the c.781A $>\mathrm{T}$ mutation using denaturing high-performance liquid chromatography, but were unable to find it (data not shown) (Lam 2006). Taken all together, these findings suggest that c.781A $>\mathrm{T}$ is the diseasecausing mutation in this family.

\section{Discussion}

Using a whole-genome scan with SNP microarrays, we have mapped the ARO locus of this ARO family to CLCN7. Interestingly, only a few cases of ARO have been ascribed to mutations in the CLCN7 gene (Cleiren et al. 2001). CLCN7 is located on the ruffled
Fig. 2 Electropherograms of exon 9 of the CLCN7 gene. Top panel The father is heterozygous for $\mathrm{c} .781 \mathrm{~A}>\mathrm{T}$. The heterozygous site is denoted by the letters $A / T$. Bottom panel The patient is homozygous for c.781A $>\mathrm{T}$. The mutation c.781A > T changes codon 261 (bracketed in the figure) from ATC to TTC - i.e., I261F. The sequences are shown in the sense direction

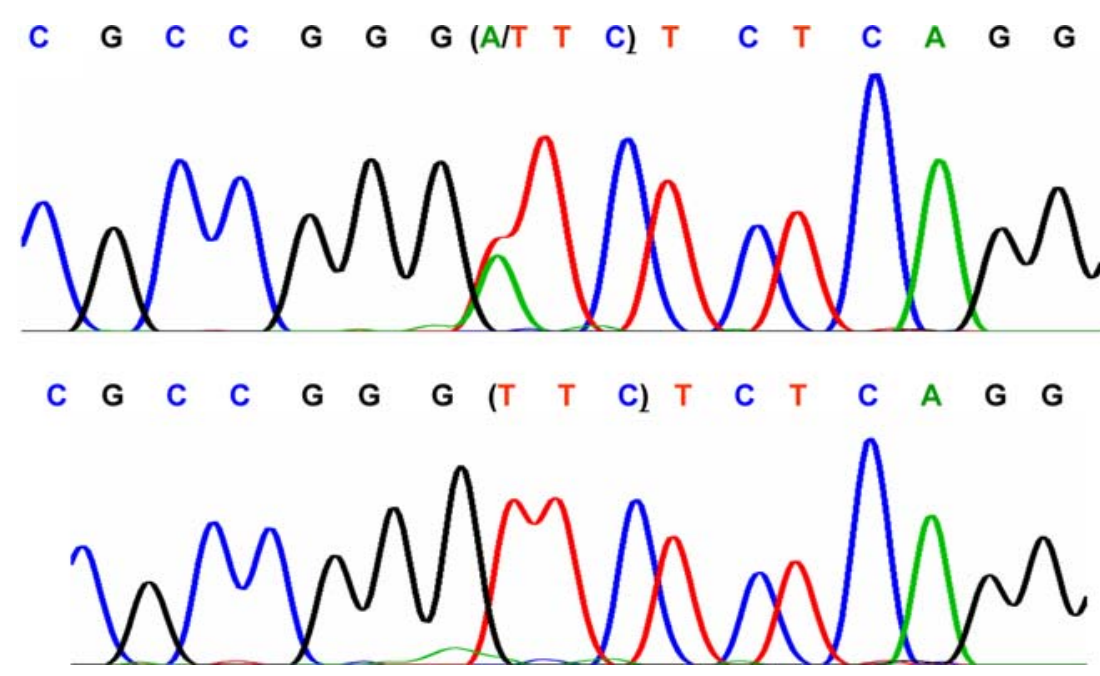


border of osteoclasts and participates in the acidification of the resorption lacuna. CLCN7 together with other chloride channel proteins form a large gene family that is found in bacteria, archae, and eukaryotes. These proteins form a homodimeric channel structure with one pore per subunit, with each subunit consisting of 18 helices (Dutzler et al. 2002). At its binding site in the pore, chloride interacts with the ends of four helices (helices D, F, N, and R) that extend out of both sides of the membrane. The chloride-coordinating alpha helices are thought to have an electrical dipole moment, thereby creating a positive potential at the binding site formed by their ends. The chloride binding site can be occupied either by a chloride ion or by a glutamate carboxyl group located at the start of helix F. Mutations of this glutamate residue in Torpedo ray $\mathrm{ClC}$ channels were found to alter the gating of the channel (Dutzler et al. 2003). The mutation, p.I261F, is located in helix F of CLCN7 and may affect the dipole moment of the helix or the coordination of chloride in the pore of the channel. Interestingly, p.P249L in helix F causes autosomal dominant osteopetrosis, type II (Cleiren et al. 2001). Because the parents in the present case are phenotypically normal, it is likely that the mutation p.I261F causes loss-of-function of the mutant channel, but has incomplete effect on the heterodimer. This mutation might be an ethnic-specific mutation as it has not been reported in other populations.

We were able to prioritize the CLCN7 loci for mutational analysis based on the history of consanguinity, by performing a whole-genome scan for homozygous chromosomal regions using SNP microarrays. Consanguinity of the parents is common in patients with ARO (Frattini et al. 2000; Kornak et al. 2001; Cleiren et al. 2001; Chalhoub et al. 2003). The mapping study reported here was essential in preparing the ground for the mutation study by decreasing the burden of completely sequencing all possible loci for this disease. The results of the mapping study and the mutation study proved to be consistent, thereby validating this approach and saving us a considerable amount of effort and time. This approach can be used to standardize molecular investigations of genetic diseases due to consanguinity to a whole-genome scan and the subsequent sequencing of the mapped disease gene. The savings in the cost of the analysis will be more if the disease has marked locus heterogeneity, such as xeroderma pigmentosum, which has eight known loci (Lam et al. 2005). With future reductions in the cost of SNP genotyping, whole-genome scans will become the standard for the diagnosis of inherited diseases due to consanguinity (Lam et al. 2005).

\section{References}

Chalhoub N, Benachenhou N, Rajapurohitam V, Pata M, Ferron M, Frattini A, Villa A, Vacher J (2003) Grey-lethal mutation induces severe malignant autosomal recessive osteopetrosis in mouse and human. Nat Med 9:399-406

Cleiren E, Benichou O, Van Hul E, Gram J, Bollerslev J, Singer FR, Beaverson K, Aledo A, Whyte MP, Yoneyama T, deVernejoul MC, Van Hul W (2001) Albers-Schönberg disease (autosomal dominant osteopetrosis, type II) results from mutations in the $\mathrm{ClCN} 7$ chloride channel gene. Hum Mol Genet 10:2861-2867

Dutzler R, Campbell EB, Cadene M, Chait BT, MacKinnon R (2002) X-ray structure of a $\mathrm{ClC}$ chloride channel at $3.0 \mathrm{~A}$ reveals the molecular basis of anion selectivity. Nature 415:287-294

Dutzler R, Campbell EB, MacKinnon R (2003) Gating the selectivity filter in ClC chloride channels. Science 300:108112

Fan JB, Chen X, Halushka MK, Berno A, Huang X, Ryder T, Lipshutz RJ, Lockhart DJ, Chakravarti A (2000) Parallel genotyping of human SNPs using generic high-density oligonucleotide tag arrays. Genome Res 10:853-860

Frattini A, Orchard PJ, Sobacchi C, Giliani S, Abinun M, Mattsson JP, Keeling DJ, Andersson AK, Wallbrandt P, Zecca L, Notarangelo LD, Vezzoni P, Villa A (2000) Defects in TCIRG1 subunit of the vacuolar proton pump are responsible for a subset of human autosomal recessive osteopetrosis. Nat Genet 25:343-346

Kornak U, Kasper D, Bosl MR, Kaiser E, Schweizer M, Schulz A, Friedrich W, Delling G, Jentsch TJ (2001) Loss of the ClC-7 chloride channel leads to osteopetrosis in mice and man. Cell 104:205-215

Lam CW (2006) Analysis of polymerase chain reaction products by denaturing high-performance liquid chromatography. Methods Mol Biol 336:73-82

Lam CW, Cheung KK, Luk NM, Chan SW, Lo KK, Tong SF (2005) DNA-based diagnosis of xeroderma pigmentosum group $\mathrm{C}$ by whole-genome scan using single-nucleotide polymorphism microarray. J Invest Dermatol 124:87-91

Shalev H, Mishori-Dery A, Kapelushnik J, Moser A, Sheffield VC, McClain A, Carmi R (2001) Prenatal diagnosis of malignant osteopetrosis in Bedouin families by linkage analysis. Penat Diagn 21:183-186 